\title{
Impact of examination stress on response time in first-year medical students
}

\author{
Singh I. ${ }^{1}$, Singh Y. ${ }^{2 *}$, Vikramaditya B. ${ }^{3}$, Singh S. ${ }^{4}$ \\ DOI: https://doi.org/10.17511/ijmrr.2020.i04.01 \\ ${ }^{1}$ Inderjit Singh, Associate Professor, Department of Physiology, Jaipur National University Institute for Medical Sciences and Research \\ Centre, Jaipur, Rajasthan, India. \\ 2* Yogesh Singh, Assistant Professor, Department of Physiology, Jaipur National University Institute for Medical Sciences and Research \\ Centre, Jaipur, Rajasthan, India. \\ ${ }^{3}$ Bibhava Vikramaditya, Assistant Professor, Department of Community Medicine, Government Medical College, Saharanpur, Uttar Pradesh, \\ India. \\ ${ }^{4}$ Santosh Singh, Department of Computer Science, Banasthali Vidyapith, Banasthali, Rajasthan, India.
}

\begin{abstract}
Background: Stress has been seen to deteriorate normal physiological functions as well as the psychological functions of the brain. Medical students face a range of enduring normative stressors in the form of academic demands. This brings upon an amount of stress on the students which is related to the examinations and concerns with achieving high grades consequently referred to as academic-related stress. Thus, it becomes crucial to set upon a tool for measuring this stress in order to present methods for overcoming it. In the present study, one such method is evaluated by assessing the reaction time of first-year medical entrants. Material and Methods: The effect of stress on response time in 50 normal healthy medical students of Sawai Man Singh Medical College, Jaipur in the age group of 18 to 21 years was explored in the study. Visual Reaction Time was recorded through the computer program. Results: There was an increase in visual reaction time for the red color in both the sexes during stressful situations as compared to stress-free situations. While a decrease in Visual Reaction Time was seen for green color. A decreased auditory reaction time for both sexes was seen in stressful situations. Conclusion: The results revealed that stress within a limit facilitates positive feedback to Central Nervous System information processing consequently decreasing the reaction time.
\end{abstract}

Keywords: Stress, State and Trait Anxiety Inventory, Central Nervous System, Visual and Auditory Reaction Time, Performance

Corresponding Author

Yogesh Singh, Assistant Professor, Department of Physiology, Jaipur National University Institute for Medical Sciences and Research Centre, Jaipur, Rajasthan, India.

Email: dryogeshsingh4u@gmail.com
How to Cite this Article

Singh I, Singh Y, Vikramaditya B, Singh S. Impact of examination stress on response time in first-year medical students. Int J Med Res Rev. 2020;8(4):288294.

Available From https://ijmrr.medresearch.in/index.php/ijmrr/article/ view/1202
To Browse

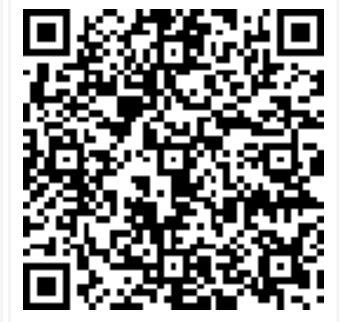

Manuscript Received 2020-07-23

Conflict of Interest No

C 2020 by Inderjit Singh, Yog Social Welfare Society. This is ar
Review Round 1 2020-08-07

Funding

$\mathrm{Nil}$
Review Round 2 2020-08-13

Ethical Approval Yes
Review Round 3

Accepted 2020-08-25

Plagiarism $X$-checker $5 \%$
Note 


\section{Introduction}

In today's time, stress has become a ubiquitous part of our lives and is common in every age. Measuring stress is difficult as there exists no definition of stress which is accepted by everyone. However, it has become clear that stress in any form affects the functioning of bodily functions either physically or mentally and hinders the wellbeing of an individual. The consequence of stress differs from individuals; it may narrow the span of attention or bring about some cognitive deficits namely irritability and a sense of helplessness. Hence it has become imperative to explore its consequences. In the biological context, stress is a mental, physical, or environmental factor causing mental or bodily tension. There has been ample talk about stress and its allied topics such as anxiety and other effects in the field of physiology since the 1950 s concerning its quantitative measurement. In the field of psychological health, the association between stress and illness has in the last decade reached a factual status [1].

It has been evident from the studies that academics and the valuations associated with it majorly examinations are a representative source of stress in students. Academics' stress in terms of performance has been studied by researchers [2, 3]. The stressful life of the medical profession begins at the student level and it is crucial to monitor the stress level of students so as to recognize the transition of a person from the state of positive stress to negative stress. it is an imperative factor in students having poor adaptability and high reactivity to stress as they are prone to potentially serious outcomes. The monitoring of stress levels of students is critically vital around their examinations, in order to recognize students having an abnormally increased level of stress who are susceptible to complications of negative stress.

In essence, this study aims to evaluate the response time during stress using examination stress as a model. The central aspect of the study is an effort so as to quantify or measure stress. The primary concern of this research is examination stress which needs some elaboration. The term "Academic Examination" refers to a heterogeneous category of events, applicable to academic examination in various disciplines namely; medical profession and professional and nonprofessional courses [4]. Due to predictability, standardization, and discreteness
Nature of academic examinations, they have been employed in studies of stress research. Academic Examination Stress may be considered as a model of "Sub-acute" stress [5]. Reaction time is the time an individual takes to respond to a stimulus. It provides an indirect index of the processing capabilities of the central nervous system as well as an uncomplicated measure to determine sensorimotor performance [6]. Response Time evaluation has the advantage of being easy, noninvasive, sensitive, and reproducible [7-9]. The aim of the present study is to evaluate the effect of examination stress on the response time of young adults. The impact of this examination concerning stress on the well being and physiological impacts on an induvial is of vital importance. Hence the current narrative evaluated stress on this front regarding the student's mental health, academic performance, and well-being.

\section{Material and Methods}

Study Setting: The present study was conducted on fifty healthy normal undergraduate first-year medical students of Sawai Man Singh Medical College, Jaipur in the age group of 18-21 years.

Study Design: The comparative cross-sectional study was conducted for a period of nine months in both examination stress and stress-free settings on 25 males along with 25 females which were selected by a simple random sampling method.

Inclusion criteria: All the subjects included in the study after written informed consent were righthanded healthy i.e. apparently free of any acute or chronic disease, with normal hearing and vision (including corrected vision) and no history of head injury, no family history of psychiatric or neurological and neuromuscular disorders in first degree relatives. The students were instructed not to alter their lifestyle or habits during the course of the study.

Exclusion criteria: Those first-year students who did not give consent to be included in the study were excluded.

Data collection procedure: After obtaining informed written consent, the subjects were asked to report in the Department of Physiology, Sawai Man Singh Medical College, Jaipur between 7-9 AM for participation in the study. The technique was explained to each and every subject and this was followed by a demonstration of its performance to 
Eliminate any kind of fear and apprehension. A demonstration and practice trial of the reaction time software was given to them to familiarize them with the software. After obtaining the personal particulars of the subject, the autonomic parameters of the subject were recorded in the supine position and psychological measures were recorded using the State-Trait Anxiety Inventory (STAI) prior to the evaluation of reaction time. The reaction times in visual and auditory domains were measured with the following design.

The subjects were seated in front of a computer screen and to put the index finger of their dominant hand lightly on the "Start" button. They were instructed to press the "Stop" button as quickly as possible when a visual/auditory stimulus was presented to them. After fifteen trials, the message "Stop" appeared on the screen.

Sensorimotor Performance was evaluated by Visual Reaction Time (VRT) and Auditory Reaction Time (ART) in millisecond (mSec.). The visual and auditory reaction time taken in cultural week served as control reading (stress-free situation) and those taken 20-30 minutes before the examination served as test readings (stressful situation). During these trials, each subject served as his/her control.

The program for measuring reaction time was prepared indigenously in the computer programming language. This program presented fifteen trials of the visual or auditory stimulus to the subject. The cues were presented with a random order or timing to minimize the bias arising from prior anticipation. After fifteen trials the message for stop appears on the screen. The software yielded an average of all the trials as the reaction time for a particular type of stimulus [10].

Surgical procedure: The study does not include any surgical procedures for measuring sensorymotor performance i.e. reaction time.

Ethical consideration and permission: The present study was conducted after receiving the ethical consideration and permission in the Department of Physiology, SMS Medical College, Jaipur, Rajasthan, India.

Statistical Analysis: Data collected was entered on an excel sheet (Microsoft Excel 2007). Continuous data were summarized in the form of mean and standard deviation. Differences in means of reaction time parameters were analyzed using the student's ' $t$ ' test.

\section{Results}

The results of the study indicate that academic pressure was the most prominent stressors. Table-1 shows Visual Reaction Time (mSec.) of Medical Entrants on various Stress-free (S-), Stressful (S+Physiology), and Anatomy (S+Anatomy) Examination Situation of Male (Table 1-a) and Female (Table 1-b) Groups for Red and Green Stimulus. Comparison of Mean VRT for Red color stimulus between Stress-free (S-) and Stressful (S+Physiology) shows that VRT for Red color was increased in both Male and Female but the increase was not significant for male and significant in female only and between Stress-free (S-) and Stressful Anatomy (S+Anatomy) indicate that VRT for Red color was increased in both Male and Females and the increase was significant for Male and Highly Significant for Females. Similarly, Comparison of Green color stimulus between Stress-free (S-) and Stressful (S+red-Physiology) shows that VRT for Green color was decreased in both the sexes but Highly Significant decrease for Male and significant for Female, however, Stress-free (S-) and Stressful Anatomy ( $\mathrm{S}+\mathrm{Green}$-Anatomy) indicate that VRT for Green color was increased in both the sexes but there was a significant increase for males and Highly Significant for females.

Table 1: Visual Reaction Time (mSec.) of Medical Entrants on various Stress-free (S-), Stressful (S+Physiology), and Anatomy (S+Anatomy) Examination Situation of male and female Groups for Red and Green Stimulus.

\begin{tabular}{|l|l|l|l|l|l|l|}
\hline \multicolumn{5}{|c|}{ Table - 1-a } & \multicolumn{5}{|c|}{ Male } \\
\hline (S-) & Mean & SD & (S+) & Mean & SD & $t$ \\
\hline S- Red & \multirow{2}{*}{0.219} & \multirow{2}{*}{0.02} & S+ Red-Physiology & 0.224 & 0.03 & 0.55 \\
\cline { 4 - 7 } & & & S+ Red-Anatomy & 0.249 & 0.04 & $0.01^{*}$ \\
\hline \multirow{2}{*}{ S- Green } & \multirow{2}{*}{0.228} & \multirow{2}{*}{0.02} & S+ Green-Physiology & 0.209 & 0.02 & $0.01^{*}$ \\
\cline { 4 - 7 } & & & S+ Green- Anatomy & 0.245 & 0.04 & $0.02^{*}$ \\
\hline
\end{tabular}

\begin{tabular}{|l|l|l|l|l|l|l|}
\hline \multicolumn{3}{|c|}{ Table - 1-b } & \multicolumn{5}{|c|}{ Female } \\
\hline S-) & Mean & SD & $($ S+) & Mean & SD & $t$ \\
\hline S- Red & 0.221 & 0.02 & S+ Red-Physiology & 0.227 & 0.01 & $0.04 *$ \\
\cline { 4 - 7 } & & & S+ Red-Anatomy & 0.262 & 0.05 & $0.01^{*}$ \\
\hline S- Green & 0.224 & 0.03 & S+ Green-Physiology & 0.214 & 0.03 & 0.07 \\
\cline { 4 - 7 } & & & S+ Green- Anatomy & 0.257 & 0.04 & $0.01^{*}$ \\
\hline
\end{tabular}

The results of Auditory Reaction Time for both the sexes are illustrated in Table 2. Auditory Reaction Time (mSec.) of Medical students on various Stressfree (S-), Stressful (S+Physiology) and Anatomy (S+Anatomy) Examination Situation of Male (Table 2 
-a) and Female Groups (Table 2-b) which shows that mean ART decreased in stressful Physiology (S+Physiology) situation and significant change was observed for both the sexes and mean ART decreased in stressful Anatomy (S+Anatomy) situation for both the sexes but no significant change was observed for both the sexes.

Table 2: Auditory Reaction Time (mSec) of Medical Students on various Stress-free (S-), Stressful (S+Physiology), and Anatomy (S+Anatomy) Examination Situation of Male and Female Groups.

\begin{tabular}{|l|l|l|l|l|l|l|}
\hline \multicolumn{3}{|c|}{ Table - 2-a } & \multicolumn{5}{|c|}{ Male } \\
\hline \multirow{3}{*}{ S- } & Mean & SD & $($ S+) & Mean & SD & $t$ \\
\cline { 2 - 7 } & 0.141 & \multirow{2}{*}{0.01} & S+Physiology & 0.126 & 0.03 & $0.04 *$ \\
\cline { 4 - 7 } & & & S+Anatomy & 0.131 & 0.02 & 0.06 \\
\hline
\end{tabular}

\begin{tabular}{|l|l|l|l|l|l|l|}
\hline \multicolumn{3}{|c|}{ Table - 2-b } & \multicolumn{5}{c|}{ Female } \\
\hline \multirow{3}{*}{ S- } & Mean & SD & S+) & Mean & SD & $t$ \\
\cline { 2 - 7 } & \multirow{2}{*}{0.145} & 0.01 & S+Physiology & 0.136 & 0.02 & $0.04 *$ \\
\cline { 4 - 7 } & & & S+Anatomy & 0.139 & 0.02 & 0.06 \\
\hline
\end{tabular}

S+ = Stressful and S- = Stressfree

Significant $=p<0.05^{*}$, Non-significant $=p>0.05$

\section{Discussion}

As indicated earlier, the study was concerned with exploring the effect of stress on selected measures which encompassed Visual Reaction Time, Auditory Reaction Time in a group of medical students. The level of stress was then evaluated employing a State and Trait Anxiety Inventory (STAI). These medical students were subjected to visual and auditory reaction time under two conditions namely a non-stress condition with no pressure of examinations and during the time when they were anticipated to appear in examinations i.e. stressful conditions.

It was seen that stress within a limit facilitates positive feedback to CNS information processing consequently decreasing the Reaction Time. While stress level exceeding the capacity of the coping mechanism of an individual adversely affects the CNS information processing consequentially, a worsening of psychomotor performance is seen.

Analyzing the VRT for red color, it was found to be high in a stressful condition for both sex groups, a statistical increase was seen in females. This aspect is comparable to the findings of Venkatesh et al [11]. VRT for red color increased in both the
S+Physiology and S+Anatomy situations as compared to that in a Stressfree (S-) situation, so observed and it could be due to an increased neural information processing time within the CNS supplanted by the inherent sympathetic stimulating and anxiety promoting effects of the presented red color stimulus. Seeing red in a stressful situation, such as examination, leads to compromised performance and associated autonomic hyperactivity and physical effect which are corroborated by the findings of Elloit and Nauert $R$ [12].

The claims of the present study are further substantiated by the results of earlier research done by Hatta et al [13] who also reported an increase in response time for red color stimulus in the condition of stress.

A decrease in VRT was seen for the green color in the condition of stress so induced by academic examination in Physiology ( $\mathrm{S}+$ Physiology) in both the genders. The decrease in reaction time observed was highly significant $(p<0.001$ ) for males and significant $(p<0.05)$ for females. The observed differential reaction time so registered for the green color in the two sets of the academic examination process, namely Anatomy and Physiology could be because of underlying unequal stress (An Anatomy Examination is considered as more stressful situation as compared to Physiology Examination) so imposed on the information processing system or mechanism in the CNS by the examinations.

Garrett et al [14] and Melton [15] also reported that the reaction time to stress response is situationspecific, wherein decreased reaction time was observed in bland stressfree situations and stressful situations increased the reaction time. Mean ART decreased significantly in the S+Physiology situation for both the sexes. This could be the result of increased alertness secondary to increase sympathoadrenal response. This could contribute to a decrease in ART.

In the present study, Reaction time to a visual stimulus is found to be longer than reaction time to an auditory stimulus in both the sexes due to the shorter length of the auditory pathway. The above reasons support the results of the present study that if stress persists for longer times. It induces a change in both psychological and physiological parameter changes of the person. Kar et al [16] evaluated the effects of examination stress on cognitive function on first-year medical students and 
Found stress to be a hampering factor.

Muley $P$ et al [17] studied the effect of stress on response time in medical students and examined the readings employing paired student " $t$ " tests. The outcomes of the study showed that stress has an effect on and increases both VRT as well as ART. In the present study, it was concluded that stress has an effect on the student's cognitive performance and acts as a naturalistic acute stressor.

Yaribeygi et al [18] in a meticulous investigation assessed the effects of stress on body functions and concluded that stress can have varied actions on the body ranging from variations in homeostasis to death or life-threatening effects. The researcher reported that in several cases the, stress or a stressful work environment was the main cause of numerous pathophysiological problems of disease. It can be viewed as a triggering or advancing factor for various diseases.

Becker et al [19] studied the time course of physiological stress response to an acute stressor. The study was in accordance with the assumption that the sympathetic nervous system activation is related to the process of the working memory through prefrontal cortex stimulation while stimulation of the hypothalamic-pituitary-adrenal axis is related to events of long term memory via interactions of the hippocampus.

The physical, cognitive, emotional, behavioral sign and symptoms of stress are more commonly seen in students around their examinations because of their already increased stress levels and the evaluation of reaction time in stressful situations, using examination stress in medical students as a model, is a useful tool to quantify and assess stress. Stress quantification is relatively cumbersome and nonspecific, and not suitable for quick assessment.

The measurement of reaction time, which is an indicator of sensory-motor performance and central nervous system processing, offers a rapid and efficient method of quantification of the level of stress. A constrained and limited sample size due to the not so willing nature of the students in participation around the time of examinations served as a limiting factor.

However, an increase in sample size along with the assessment of some other autonomic functions which are affected by stress, new insights could be explored giving a better perspective.

\section{Conclusion}

The stressful life of the medical profession begins at the student level and it is imperative to monitor the stress level of students in order to identify the transition of a person from the state of positive stress to negative stress. This is especially important in students who have poor adaptability and higher reactivity to stress and therefore, are prone to potentially serious outcomes.

The monitoring of stress levels of students is critically important around their examinations, to identify students with abnormally increased stress levels who are susceptible to complications of negative stress. The present study demonstrates that stress within a limit provides positive feedback to Central Nervous System information processing thus increasing the efficiency of a person which was evident from the decrease in reaction time observed.

Whereas when the stress surpasses the capacity of a coping mechanism for a person, it adversely affects the Central Nervous System processing system which was evident from the increase in reaction time.

Stress can be considered as a ubiquitous part of every individual's life experienced at some part point in the form of acute or chronic stress. Speaking in terms of academic life, examination stress and students go hand in hand.

This aspect is vital and should be quantified for overcoming stress which otherwise could have an adverse effect on both the academic performance and cope up with the responses of physiological functions.

Future scope of the present study exhibits that exam stress affects the cognitive performance of the students and counseling and regular relaxation therapy such as yoga and meditation may be counter that stress. Hence, studies involving the relationships between relaxation techniques and stress have to be evaluated in similar settings.

\section{What does the study add to the existing knowledge?}

The present study provides a better perspective on the relationship between examination stress and sensory-motor performance in young adults while emphasizing the need for coping with the stress. 


\section{Author's contributions}

Dr. Inderjit Singh: Reviewed the draft manuscript and guided in the finalization of the manuscript.

Dr. Yogesh Singh: Concept, study design, data collection, manuscript preparation

Dr, Bibhava Vikramaditya: Statistical analysis, manuscript preparation

Mr. Santosh Singh: Developed the software for measuring the visual and auditory reaction time

\section{Acknowledgment}

The authors are grateful to all the participants for contributing their time to conduct the present study. The authors extend heartiest thanks to the Department of Physiology, Sawai Man Singh Medical College, Jaipur for their support throughout the study.

\section{Reference}

01. Pollock K. On the nature of social stressProduction of a modern mythology. Soc Sci Med. 1988;26(3)381-392.

doi: $\quad 10.1016 / 0277-9536(88) 90404-2 \quad$ [Crossref]

02. Akgun S, Ciarrochi J. Learned Resourcefulness Moderates the Relationship Between Academic Stress and Academic Performance. Educ Psychol. 2003;23(3)287-294.

doi: $10.1080 / 0144341032000060129$ [Crossref]

03. Struthers CW, Perry RP, Menec VH. An examination of the relationship among academic stress, coping, motivation, and performance in college. Res High Educ. 2000;41(5)581-592.

doi: 10.1023/A:1007094931292 [Crossref]

04. Stowell JR. Use and Abuse of Academic Examinations in Stress Research. Psychosom Med. 2003;65(6)1055-1057.

doi: $\quad$ 10.1097/01.PSY.0000097349.84109.1F [Crossref]

05. Kiecolt-Glaser JK, Garner W, Speicher C, Penn GM, Holliday J, Glaser R. Psychosocial modifiers of immunocompetence in medical students. Psychosom Med. 1984;46(1)7-14. doi: 10.1097/00006842-198401000-00003 [Crossref]
06. Kar M. Evaluation of Examination Stress and Its Effect on Cognitive Function among First Year Medical Students. J Clin Diagnostic Res. 2014;8(8)BC05.

doi: $\quad 10.7860 /$ JCDR/2014/9014.4680 [Crossref]

07. Shavanani AB, Udupa KA. Acute effect of Mukh bhastrika (a yogic bellows type breathing) on reaction time. Indian J Physiol Pharmacol. 2003;47;297-300.

[Crossref]

08. Malathi A, Parulkar VG. Evaluation of anxiety status in medical students prior to examination stress. Indian J Physiol Pharmacol. 1992;36;121-122.

[Crossref]

09. Malathi A, Parulkar VG. Effect of yogasanas on the visual and auditory reaction time. Indian $\mathrm{J}$ Physiol Pharmacol. 1989;33(12):110-112.

[Crossref]

10. Zajdel R, Nowak D. Simple and complex reaction time measurement- a preliminary evaluation of new approach and diagnostic tool. Comput Biol Med. 2007;37(12)1724-1730.

doi: 10.1016/j.compbiomed.2007.04.008 [Crossref]

11. Venkatesh D, Ramachandra DL, Baboo NS, Rajan BK. Impact of psychological stress, gender and colour on visual response latency. Indian J Physiol Pharmacol. 2002;46(3)333-337. [Crossref]

12. Elliot AJ, Niesta D. Romantic red- Red enhances men's attraction to women. J Personal Social Psychol. 2008;95(5)1150.

[Crossref:Crossref]

13. Hatta T, Yoshida H, Kawakami A, Okamoto M. Color of Computer Display Frame in Work Performance, Mood, and Physiological Response. Percept Mot Skills. 2002;94(1)39-46. doi: $10.2466 /$ pms.2002.94.1.39 [Crossref]

14. Melton AW. The methodology of experimental studies of human learning and retention I- The functions of a methodology and the available criteria for evaluating different experimental methods. Psychol Bull. 1936;33(5)305-394.

doi: $10.1037 / \mathrm{h} 0055784$

[Crossref:https://doi.org/10.1037/h0055784] 
15. Lofthus GK. Sensorimotor Performance and Limb Preference. Percept Mot Skills. $1981 ; 52(3) 683-693$.

doi: $10.2466 / p m s .1981 .52 .3 .683$ [Crossref]

16. Muley DA, Wadikar SS, Muley DP. Effect of exam Stress on reaction time in medical students. Indian J Basic Appl Med Res. 2016;5(4)733-739. [Crossref]

17. Garrett HE. The two-factor theory and its criticism. Psychol Rev. 1935;42;293-301.

doi: 10.1037/h0055118 [Crossref]
18. Becker L, Rohleder N. Time course of the physiological stress response to an acute stressor and its associations with the primacy and recency effect of the serial position curve. PLoS One. $2019 ; 14(5)$ e0213883.

doi: $10.1371 /$ journal.pone.0213883

19. Yaribeygi $H$, Panahi $Y$, Sahraei $H$, Johnston TP, Sahebkar A. The impact of stress on body function- A review. EXCLI J. 2017;16;10571072.

doi: $10.17179 /$ excli2017-480 [Crossref] 